\title{
COVID-19 Vaccine-Related Attitudes and Beliefs in Canada: National Cross-sectional Survey and Cluster Analysis
}

Jamie L Benham ${ }^{1,2}$, MD, PhD; Omid Atabati ${ }^{3}$, MSc; Robert J Oxoby ${ }^{3}, \mathrm{PhD}$; Mehdi Mourali ${ }^{4}$, PhD; Blake Shaffer ${ }^{3}$, $\mathrm{PhD}$; Hasan Sheikh ${ }^{5}$, MPA, MD; Jean-Christophe Boucher ${ }^{6}$, PhD; Cora Constantinescu ${ }^{2}$, MSc, MD; Jeanna Parsons Leigh $^{7}, \mathrm{PhD}$; Noah M Ivers ${ }^{8}$, MD, PhD; Scott C Ratzan ${ }^{9}$, MPA, MA, MD; Madison M Fullerton ${ }^{2}$, MSc; Theresa Tang $^{2}$, BHSc; Braden J Manns ${ }^{1,2}$, MSc, MD; Deborah A Marshall ${ }^{1,2}, \mathrm{PhD}$; Jia Hu ${ }^{2 *}$, MD; Raynell Lang ${ }^{1 *}$, MSc, MD

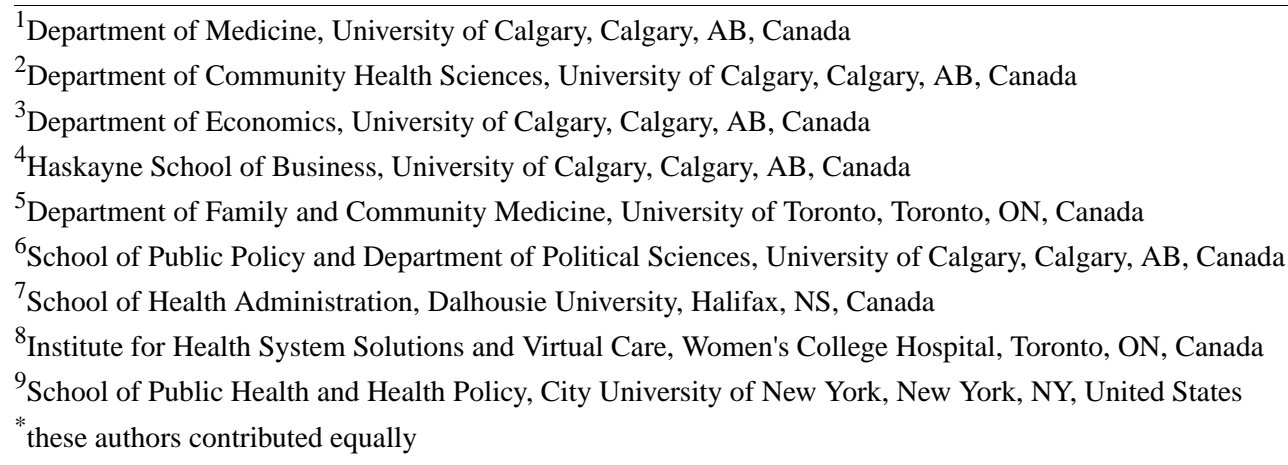

Corresponding Author:

Jamie L Benham, MD, PhD

Department of Medicine

University of Calgary

1820 Richmond Road SW

Calgary, AB, T2T5C7

Canada

Phone: 14039558987

Email: jlbenham@ucalgary.ca

\section{Abstract}

Background: There are concerns that vaccine hesitancy may impede COVID-19 vaccine rollout and prevent the achievement of herd immunity. Vaccine hesitancy is a delay in acceptance or refusal of vaccines despite their availability.

Objective: We aimed to identify which people are more and less likely to take a COVID-19 vaccine and factors associated with vaccine hesitancy to inform public health messaging.

Methods: A Canadian cross-sectional survey was conducted in Canada in October and November 2020, prior to the regulatory approval of the COVID-19 vaccines. Vaccine hesitancy was measured by respondents answering the question "what would you do if a COVID-19 vaccine were available to you?" Negative binomial regression was used to identify the factors associated with vaccine hesitancy. Cluster analysis was performed to identify distinct clusters based on intention to take a COVID-19 vaccine, beliefs about COVID-19 and COVID-19 vaccines, and adherence to nonpharmaceutical interventions.

Results: Of 4498 participants, 2876 (63.9\%) reported COVID-19 vaccine hesitancy. Vaccine hesitancy was significantly associated with (1) younger age (18-39 years), (2) lower education, and (3) non-Liberal political leaning. Participants that reported vaccine hesitancy were less likely to believe that a COVID-19 vaccine would end the pandemic or that the benefits of a COVID-19 vaccine outweighed the risks. Individuals with vaccine hesitancy had higher prevalence of being concerned about vaccine side effects, lower prevalence of being influenced by peers or health care professionals, and lower prevalence of trust in government institutions.

Conclusions: These findings can be used to inform targeted public health messaging to combat vaccine hesitancy as COVID-19 vaccine administration continues. Messaging related to preventing COVID among friends and family, highlighting the benefits, emphasizing safety and efficacy of COVID-19 vaccination, and ensuring that health care workers are knowledgeable and supported in their vaccination counselling may be effective for vaccine-hesitant populations. 
(JMIR Public Health Surveill 2021;7(12):e30424) doi: 10.2196/30424

\section{KEYWORDS}

coronavirus; COVID-19; public health; marketing; behavior; risk reduction; attitudes; compliance; vaccine; hesitancy; risk; belief; communication; cross-sectional; Canada; gender; education; income; race; ethnicity

\section{Introduction}

In the fall of 2020, regions of Canada were experiencing a second wave of COVID-19 with rising case counts, hospitalizations, and deaths [1]. Although several vaccines against SARS-CoV-2 were in development [2], they were not yet available as public health tools to mitigate the spread of COVID-19, as the first COVID-19 vaccine was not authorized by Health Canada until December 9, 2020 [2]. This meant that in the fall of 2020, nonpharmacologic interventions (NPIs), including practicing physical distancing, wearing a face mask if physical distancing is not possible, staying home when sick, and limiting large gatherings were the only means to reduce the transmission of COVID-19 [3].

Although there was great optimism about the potential emergence of safe and effective vaccines against SARS-CoV-2, COVID-19 vaccine hesitancy was becoming evident in the summer and fall of 2020 [4-7]. Vaccine hesitancy is defined by the Strategic Advisory Group of Experts (SAGE) Working Group as a "delay in acceptance or refusal of vaccination despite availability of vaccination services" [8]. The reasons for vaccine hesitancy are heterogeneous and complex [9]. The SAGE Working Group created a framework of vaccine hesitancy determinants, which consists of 3 domains: (1) contextual influences (eg, socioeconomic group, political climate), (2) individual and social group influences (eg, social norm, personal experience), and (3) vaccine characteristics (eg, perceived risks and benefits, health care provider attitudes) [8]. This framework can be used to determine the potential factors contributing to vaccine hesitancy with respect to a COVID-19 vaccine within the unique context of the COVID-19 pandemic.

Several studies have looked at the risk factors for vaccine hesitancy in populations around the world and have found that many different factors, including sociodemographic variables and concerns about efficacy and safety of COVID-19 vaccines, may contribute to COVID-19 vaccine hesitancy [10]. It has been noted, however, that factors associated with vaccine hesitancy identified in the general population may not be consistent with factors associated with vaccine hesitancy in specific subpopulations [11]. Therefore, to improve overall vaccine uptake, it is important to examine the risk factors for vaccine hesitancy in the specific population segments who report increased vaccine hesitancy.

In the summer of 2020, we designed a mixed methods study to examine COVID-19 attitudes, beliefs, and behaviors among Canadians with an overreaching goal of informing targeted public health messaging to improve adherence to NPIs and vaccine uptake. We have previously published the initial phases of this mixed methods study including a pilot survey [5] and a qualitative study [4]. This preliminary work found that there were mixed views regarding willingness to take a COVID-19 vaccine and identified a number of risk factors with respect to COVID-19 vaccine hesitancy, including low perceived risk of COVID-19 infection, vaccine-specific concerns, low adherence to NPIs, and sources of COVID-19 information [4,5].

Based on the findings from the initial work in our mixed methods study $[4,5]$, we designed a national survey to further explore the risk factors for vaccine hesitancy to identify segmented populations of individuals with vaccine hesitancy to inform targeted public health messaging campaigns. The objectives of this study were to (1) identify which groups of people are more or less likely to take a COVID-19 vaccine among Canadian adults, (2) determine which attitudes toward COVID-19 are associated with vaccine hesitancy, (3) determine if vaccine hesitancy is associated with adherence to NPIs for COVID-19, and (4) evaluate the relationship between persons' vaccine attitudes and their sources of COVID-19 information.

\section{Methods}

\section{Study Design, Participants, and Setting}

We used a cross-sectional survey to assess the attitudes and beliefs about vaccines and vaccine hesitancy among adults aged 18 years or older living in Canada. The survey was administered online by the Angus Reid Institute [12], a national, not-for-profit, research foundation, from October 27 to November 2, 2020, as the preliminary data on vaccine efficacy and safety of the COVID-19 vaccine, Pfizer-BioNTech, was being submitted to Health Canada for review but before it was approved for use in Canada on December 9, 2020. Survey participants were drawn randomly from the Angus Reid Forum and contacted electronically. The Angus Reid Forum is comprised of 70,000 individuals from across Canada designed to be representative of the Canadian population with sociodemographic characteristics verified to match electoral and census data in each sampling region [12]. To obtain a sample size of 4500 , the survey was distributed to 14,887 potential participants. Sampling was stratified for equal representation of Alberta residents and residents of the other Canadian provinces combined. This sampling strategy was used to allow for comparison of 2 Canadian applications used to facilitate contact tracing, that is, ABTraceTogether (a contact tracing application, which is only available in Alberta) and COVID Alert (an exposure notification application available in 8 provinces and the Northwest territories). A copy of the survey questions that were administered can be found in Multimedia Appendix 1.

This study was approved by the Conjoint Health Research Ethics Board at the University of Calgary (REB20-1228). Informed consent was obtained from each participant prior to commencing the survey, and participation was voluntary. Responses were deidentified at the time of collection to ensure participant anonymity and privacy. If participants started the survey but 
did not complete it, it was assumed that consent was withdrawn and their survey responses were not saved. Consistent with Angus Reid Forum policy [12], members of the Angus Reid Forum who completed the survey received a small monetary incentive. The Strengthening Reporting of Observational Studies in Epidemiology (STROBE) checklist was used to report our findings [13].

\section{Outcome Measure}

The main outcome measure was vaccine hesitancy. Survey participants were asked what they would do if a COVID-19 vaccine were available to them and given the following 4 options: (1) get a vaccine as soon as possible, (2) eventually get a vaccine, but wait a while first, (3) not get a vaccine, or (4) not sure. Vaccine hesitancy was defined as any of the latter 3 responses consistent with the SAGE Working Group definition of vaccine hesitancy [8].

\section{Risk Factors for Vaccine Hesitancy}

We considered factors that could be associated with vaccine hesitancy in each of the domains of the SAGE framework (contextual influences, individual and group influences, and vaccine-specific factors) [8] based on a review of the literature, focus groups [4], and a pilot survey [5] that we completed in Alberta, Canada in the summer of 2020. For contextual influences, we determined demographic factors, including sex, age, geographical region, household income, highest level of education, ethnicity, and political leaning. In terms of individual and group influences, we determined participants' attitudes toward COVID-19 and the COVID-19 vaccine, adherence to NPIs (ie, physical distancing, masking, reducing interactions with others, staying home when sick), trusted sources of COVID-19 information, and trusted institutions. For vaccine characteristics, participants were asked about the perceived risks and benefits of COVID-19 vaccines.

\section{Statistical Analysis}

Descriptive statistics (percentage frequencies) were calculated for all participant characteristics, adherence to NPIs, attitudes toward COVID-19 and COVID-19 vaccines, and trusted sources of COVID-19 information. Respondents were excluded if they did not answer all survey questions, and therefore, there were no missing data. Negative binomial regression models were used to estimate crude prevalence ratios (PRs) for factors associated with being vaccine hesitant compared to not being vaccine hesitant. Each PR was reported with the associated 95\%
CI. We used multiple models to examine the association between vaccine hesitancy and each of the following: (1) sociodemographic characteristics, (2) attitudes toward COVID-19 vaccine, (3) adherence toward NPIs, (4) attitudes toward COVID-19, and (5) trusted sources of COVID-19 information. We also calculated adjusted prevalence ratios (aPRs) by using sociodemographic characteristics identified through a literature search $[10,14-16]$ as being associated with vaccine hesitancy, including sex at birth, age, ethnicity, province of residence, education, household income, and political leaning.

To identify data-driven patterns in survey responses with respect to vaccine hesitancy, we used cluster analysis. The cluster analysis was based on intention to take a COVID-19 vaccine, beliefs about COVID-19 and COVID-19 vaccine, and adherence to NPIs. The K-means algorithm was used to partition the data set into distinct clusters. This iterative algorithm assigns observations to a cluster such that within each cluster, the sum of the squared distance between observations and the arithmetic mean of all observations is minimized. Cluster analysis was used to integrate COVID-19 vaccine intention, COVID-19 beliefs, and adherence to NPIs into similar like-minded groupings to identify insights that can be utilized for targeted messaging and interventions. By using several exposures to establish these clusters, we aimed to create clusters with greater similarity in motivations and attitudes for vaccine intention and gain a deeper understanding of vaccine hesitancy. Negative binomial regression was used to estimate crude PRs and 95\% CI comparing sociodemographic characteristics between each of the clusters with cluster 2 as the reference. Analyses were conducted using STATA Version 15.1 (Stata Corp). A $P$ value of $<.05$ was set as significant.

\section{Results}

\section{Survey Participation}

Of the 14,887 survey invitations distributed, 5893 (39.6\%) invitations were accepted in the 7 days the survey was available. Of those, $4498(76.3 \%)$ participants completed the survey and were included in the analysis (Table 1), while 1395 (23.7\%) participants were excluded owing to one or more incomplete responses. Participants who completed the survey were similar to those who started but did not complete the survey in terms of sex, age, province of residence, highest level of education, and ethnicity. 
Table 1. Participant characteristics and association with COVID-19 vaccine hesitancy in October to November 2020 (N=4498).

\begin{tabular}{|c|c|c|c|c|c|}
\hline \multirow[b]{2}{*}{ Characteristic } & \multirow[b]{2}{*}{ Total, n (\%) } & \multicolumn{2}{|c|}{ Vaccine hesitancy, n (\%) } & \multirow[t]{2}{*}{ Prevalence ratio $^{\mathrm{a}}(95 \% \mathrm{CI})$} & \multirow[t]{2}{*}{ Adjusted prevalence ratio $^{\mathrm{b}}(95 \% \mathrm{CI})$} \\
\hline & & No & Yes & & \\
\hline Participants & $4498(100)$ & $1622(36.1)$ & $2876(63.9)$ & $\mathrm{N} / \mathrm{A}^{\mathrm{c}}$ & N/A \\
\hline \multicolumn{6}{|l|}{ Sex at birth } \\
\hline Female & $2294(51)$ & $815(35.5)$ & $1479(64.5)$ & $\operatorname{Ref}^{d}$ & Ref \\
\hline Male & $2204(49)$ & 807 (36.6) & $1397(63.4)$ & $0.98(0.91-1.06)$ & $0.93(0.86-1.01)$ \\
\hline \multicolumn{6}{|l|}{ Age (years) } \\
\hline $18-34$ & $1341(29.8)$ & $505(37.7)$ & $836(62.3)$ & Ref & Ref \\
\hline $35-54$ & $1585(35.2)$ & $504(31.8)$ & $1081(68.2)$ & $1.09(1.00-1.20)$ & $1.04(0.95-1.14)$ \\
\hline $55+$ & $1572(35)$ & $613(39)$ & $959(61)$ & $0.98(0.89-1.07)$ & $0.90(0.82-0.99)$ \\
\hline \multicolumn{6}{|l|}{ Province of residence } \\
\hline Alberta & $1998(44.4)$ & $672(33.6)$ & $1326(65.4)$ & Ref & Ref \\
\hline British Columbia & $502(11.2)$ & $176(35.1)$ & $326(64.9)$ & $0.98(0.87-1.10)$ & $1.04(0.92-1.17)$ \\
\hline Prairie provinces ${ }^{\mathrm{e}}$ & $445(9.9)$ & $156(35.1)$ & $259(58.2)$ & $0.98(0.76-1.11)$ & $0.95(0.84-1.08)$ \\
\hline Ontario & $800(17.8)$ & $311(38.9)$ & $489(61.1)$ & $0.92(0.83-1.02)$ & $0.96(0.87-1.07)$ \\
\hline Quebec & $502(11.2)$ & $203(40.4)$ & $299(59.6)$ & $0.90(0.79-1.02)$ & $0.97(0.85-1.10)$ \\
\hline Atlantic provinces ${ }^{\mathrm{e}}$ & $251(5.6)$ & $104(41.4)$ & $147(58.6)$ & $0.88(0.74-1.05)$ & $0.95(0.80-1.13)$ \\
\hline \multicolumn{6}{|l|}{ Household income $^{f}$ (CAD) } \\
\hline$<\$ 50,000$ & $1030(22.9)$ & $342(33.2)$ & $688(66.8)$ & Ref & Ref \\
\hline$\$ 50,000-\$ 99,999$ & $1353(30.1)$ & $486(35.9)$ & $867(64.1)$ & $0.96(0.87-1.06)$ & $0.97(0.88-1.08)$ \\
\hline$\$ 100,000-\$ 199,999$ & $1300(28.9)$ & $511(39.3)$ & 789 (60.7) & $0.91(0.82-1.01)$ & $0.93(0.84-1.04)$ \\
\hline$\geq \$ 200,000$ & $229(5.1)$ & $102(44.5)$ & $127(55.5)$ & $0.83(0.69-1.00)$ & $0.85(0.70-1.03)$ \\
\hline Rather not say & $586(13)$ & $181(30.9)$ & $405(69.1)$ & $1.03(0.92-1.17)$ & $1.02(0.90-1.15)$ \\
\hline \multicolumn{6}{|l|}{ Highest level of education } \\
\hline High school graduate or less & 897 (19.9) & $256(28.5)$ & $641(71.5)$ & Ref & Ref \\
\hline Some college or trade school & $840(18.7)$ & $240(28.6)$ & $600(71.4)$ & $1.00(0.89-1.12)$ & $1.01(0.90-1.13)$ \\
\hline College or trade school & $996(22.1)$ & $301(30.2)$ & $695(69.8)$ & $0.98(0.88-1.09)$ & $0.98(0.88-1.10)$ \\
\hline Some university & $454(10.1)$ & $185(40.7)$ & $269(59.3)$ & $0.83(0.72-0.96)$ & $0.85(0.73-0.97)$ \\
\hline University degree & $1311(29.1)$ & $640(48.8)$ & $671(51.2)$ & $0.72(0.64-0.80)$ & $0.73(0.65-0.81)$ \\
\hline \multicolumn{6}{|l|}{ Ethnicity } \\
\hline Caucasian & $3862(85.9)$ & $1430(37)$ & $2432(63)$ & Ref & Ref \\
\hline Indigenous/First Nations/Metis/Inuit & $228(5)$ & $68(29.8)$ & $160(70.2)$ & $1.11(0.95-1.31)$ & $1.09(0.93-1.27)$ \\
\hline Asian & $193(4.3)$ & $65(33.7)$ & $128(66.3)$ & $1.05(0.88-1.26)$ & $1.15(0.96-1.37)$ \\
\hline Caribbean/African/South American & $70(1.6)$ & $19(27.1)$ & $51(72.9)$ & $1.16(0.88-1.53)$ & $1.16(0.88-1.54)$ \\
\hline Other & $145(3.2)$ & $40(27.6)$ & $105(72.4)$ & $1.15(0.95-1.40)$ & $1.10(0.91-1.34)$ \\
\hline \multicolumn{6}{|l|}{ Political leaning } \\
\hline Liberal & $1841(40.9)$ & $936(50.8)$ & $905(49.2)$ & $0.73(0.66-0.80)$ & $0.74(0.67-0.82)$ \\
\hline Moderate/middle of the road & $1029(22.9)$ & $334(32.5)$ & $695(67.5)$ & Ref & Ref \\
\hline Conservative & $1628(36.2)$ & $352(21.6)$ & $1276(78.4)$ & $1.16(1.06-1.27)$ & $1.18(1.07-1.29)$ \\
\hline
\end{tabular}

${ }^{a}$ Prevalence ratio is the prevalence of vaccine hesitancy compared with the prevalence of planning to take a COVID-19 vaccine.

${ }^{\mathrm{b}}$ Adjusted for sex, age, province of residence, household income, education level, ethnicity, and political leaning.

${ }^{\mathrm{c}}$ N/A: not applicable. 
${ }^{\mathrm{d}}$ Ref: reference value.

${ }^{\mathrm{e}}$ Prairie provinces include Saskatchewan and Manitoba; Atlantic provinces include Nova Scotia, New Brunswick, Prince Edward Island, and Newfoundland and Labrador.

${ }^{\mathrm{f}} \mathrm{CAD} \$ 1=\mathrm{US} \$ 0.75$.

\section{Participant Characteristics}

Participant demographic and socioeconomic characteristics are presented in Table 1. The majority of the participants were females $(2294 / 4498,51 \%)$ and Caucasian $(3862 / 4498,85.9 \%)$. The mean participant age was 47 (SD 16) years with participant ages ranging from 18 to 94 years. The majority of the participants indicated that they were vaccine hesitant and reported they would delay taking a COVID-19 vaccine when offered one $(1817 / 4498,40.4 \%)$, not take a COVID-19 vaccine (708/4498, 15.7\%), or were not sure about taking a COVID-19 vaccine $(351 / 4498,7.8 \%)$. The remaining one-third (1622/4498, $36.1 \%$ ) of the participants reported that they would take a COVID-19 vaccine as soon as possible. Participants aged 55 years or older had lower prevalence of vaccine hesitancy compared with those aged 18-34 years (aPR 0.90, 95\% CI 0.82-0.99). University education was also associated with lower prevalence of vaccine hesitancy. Compared with participants who reported their highest level of education as high school graduate or less, the adjusted prevalence was 0.85 (95\% CI $0.73-0.97)$ for some university education and 0.73 (95\% CI 0.65-0.81) for participants who had completed a university degree. Liberal political leaning was associated with lower prevalence of vaccine hesitancy compared with participants who reported moderate or middle of the road political leaning (aPR 0.74, 95\% CI 0.67-0.82), while conservative political leaning was associated with higher prevalence of vaccine hesitancy (aPR 1.18, 95\% CI 1.07-1.29). Biological sex, household income, ethnicity, and province of residence were not associated with vaccine hesitancy.

\section{Attitudes Toward COVID-19 Vaccine}

More than half of the participants $(2501 / 4498,55.6 \%)$ felt that the benefits of taking a vaccine outweigh its risks, while 969 $(22 \%)$ were unsure and $1028(22 \%)$ disagreed (Table 2). 
Table 2. Associations between COVID-19 vaccine hesitancy and attitudes toward COVID-19 vaccines in October to November 2020 (N=4498).

\begin{tabular}{llllll} 
& Total, n (\%) & \multicolumn{2}{c}{ Vaccine hesitancy, n (\%) } & Prevalence ratio ${ }^{\mathrm{a}}$ (95\% CI) & Adjusted prevalence ratio ${ }^{\mathrm{b}}(95 \% \mathrm{CI})$ \\
\hline Participants & $4498(100)$ & No & Yes & N/A
\end{tabular}

Attitudes toward COVID-19 vaccines

Would take a vaccine to protect family

\begin{tabular}{llllll} 
Agree & $3293(73.2)$ & $1603(48.7)$ & $1690(51.3)$ & $\operatorname{Ref}^{\mathrm{d}}$ & \multicolumn{2}{l}{$\operatorname{Ref}$} \\
Disagree & $810(18)$ & $17(2.1)$ & $793(97.9)$ & $1.91(1.75-2.08)$ & $1.77(1.62-1.94)$ \\
Not sure & $395(8.8)$ & $2(0.5)$ & $393(99.5)$ & $1.94(1.74-2.16)$ & $1.85(1.66-2.07)$
\end{tabular}

$A$ vaccine will end the pandemic

$\begin{array}{llllll}\text { Agree } & 1265(28.1) & 691(54.6) & 574(45.4) & \text { Ref } & \text { Ref } \\ \text { Disagree } & 2214(49.2) & 587(26.5) & 1627(73.5) & 1.62(1.47-1.78) & 1.54(1.40-1.70) \\ \text { Not sure } & 1019(22.7) & 344(33.8) & 675(66.2) & 1.46(1.31-1.63) & 1.43(1.28-1.61)\end{array}$

Usually get the flu vaccine

$\begin{array}{llllll}\text { Agree } & 2523(56.1) & 1301(51.6) & 1222(48.4) & \text { Ref } & \text { Ref } \\ \text { Disagree } & 1901(42.3) & 301(15.8) & 1600(84.2) & 1.74(1.61-1.87) & 1.67(1.55-1.80) \\ \text { Not Sure } & 74(1.6) & 20(27) & 54(73) & 1.51(1.15-1.98) & 1.50(1.14-1.97)\end{array}$

\section{Concern about short-term side effects}

$\begin{array}{llllll}\text { Agree } & 2583(57.4) & 533(20.6) & 2050(79.4) & \text { Ref } & \text { Ref } \\ \text { Disagree } & 1443(32.1) & 922(63.9) & 521(36.1) & 0.45(0.41-0.50) & 0.47(0.43-0.52) \\ \text { Not Sure } & 472(10.5) & 167(35.4) & 305(64.6) & 0.81(0.72-0.92) & 0.83(0.73-0.93)\end{array}$

\section{Concern about long-term side effects}

$\begin{array}{llllll}\text { Agree } & 2703(60.1) & 542(20.1) & 2161(79.9) & \text { Ref } & \text { Ref } \\ \text { Disagree } & 1294(28.8) & 881(68.1) & 413(31.9) & 0.40(0.36-0.44) & 0.42(0.38-0.46) \\ \text { Not sure } & 501(11.1) & 199(39.7) & 302(60.3) & 0.75(0.67-0.85) & 0.78(0.69-0.88)\end{array}$

Vaccine developed too fast

$\begin{array}{llllll}\text { Agree } & 1985(44.1) & 162(8.2) & 1823(91.8) & \text { Ref } & \text { Ref } \\ \text { Disagree } & 1874(41.7) & 1248(66.6) & 626(33.4) & 0.36(0.33-0.40) & 0.38(0.35-0.42) \\ \text { Not sure } & 639(14.2) & 212(33.2) & 427(66.8) & 0.73(0.65-0.81) & 0.75(0.67-0.83)\end{array}$

Vaccine benefits outweigh the risks

$\begin{array}{llllll}\text { Agree } & 2501(55.6) & 1457(58.3) & 1044(41.7) & \text { Ref } & \text { Ref } \\ \text { Disagree } & 1028(22.9) & 40(3.9) & 988(96.1) & 2.30(2.11-2.51) & 2.17(1.98-2.38) \\ \text { Not sure } & 969(21.5) & 125(12.9) & 844(87.1) & 2.09(1.91-2.28) & 2.02(1.85-2.22)\end{array}$

Would take vaccine if family/friends do

$\begin{array}{llllll}\text { Agree } & 1681(37.4) & 745(44.3) & 936(55.7) & \text { Ref } & \text { Ref } \\ \text { Disagree } & 2296(51) & 717(31.2) & 1579(68.8) & 1.24(1.14-1.34) & 1.17(1.08-1.27) \\ \text { Not sure } & 521(11.6) & 160(30.7) & 361(69.3) & 1.24(1.10-1.41) & 1.21(1.07-1.37)\end{array}$

Would take vaccine if advised by family doctor/pharmacist/public health official

$\begin{array}{llllll}\text { Agree } & 2775(61.7) & 1422(51.2) & 1353(48.8) & \text { Ref } & \text { Ref } \\ \text { Disagree } & 1319(29.3) & 131(10) & 1188(90.1) & 1.85(1.71-2.00) & 1.73(1.59-1.87) \\ \text { Not sure } & 404(9) & 69(17.1) & 335(82.9) & 1.70(1.51-1.92) & 1.65(1.46-1.86)\end{array}$

${ }^{\text {a }}$ Prevalence ratio is the prevalence of vaccine hesitancy compared with the prevalence of planning to take a COVID-19 vaccine.

${ }^{\mathrm{b}}$ Adjusted for sex, age, province of residence, household income, education level, ethnicity, and political leaning. 
${ }^{\mathrm{c}} \mathrm{N} / \mathrm{A}$ : not applicable.

${ }^{\mathrm{d}}$ Ref: reference value.

Those who disagreed had higher prevalence of vaccine hesitancy compared with those who agreed (aPR 2.17, 95\% CI 1.98-2.38; Table 2). Opinions were mixed on whether a COVID-19 vaccine would end the pandemic with $1265(28.1 \%$ ) in agreement, 2214 $(49.2 \%)$ in disagreement, and $1019(22.7 \%)$ being undecided. Participants who disagreed that the vaccine would end the pandemic had a higher prevalence of vaccine hesitancy than those who agreed (aPR 1.54, 95\% CI 1.40-1.70).

Participants reported that they would be more likely to take a COVID-19 vaccine if it was recommended by a family doctor, pharmacist, or public health nurse $(2775 / 4498,61.7 \%)$ or if their friends or family took a vaccine $(1681 / 4498,37.4 \%)$. However, the prevalence of vaccine hesitancy was higher in participants who disagreed that they would take a vaccine if their friends/family do (aPR $1.17,95 \%$ CI 1.08-1.27) or if it was recommended by a family doctor, pharmacist, or public health nurse (aPR 1.73, 95\% CI 1.59-1.87). Numerous participants $(3293 / 4498,73.2 \%)$ said they would take a COVID-19 vaccine to protect their family; participants who disagreed with this statement had a higher prevalence of vaccine hesitancy compared with those who agreed (aPR 1.77, 95\% CI 1.62-1.94). Many participants were concerned about the short-term side effects $(2583 / 4498,57.4 \%)$ and long-term side effects $(2703 / 4498,60.1 \%)$. Participants $(1874 / 4498,41.7 \%)$ who disagreed with the statement that vaccines were developed too fast had a lower prevalence of vaccine hesitancy compared with those who agreed (aPR 0.38, 95\% CI 0.35-0.42).

\section{NPIs}

The majority of the participants reported physical distancing (3782/4498, 84.1\%), wearing face masks $(3873 / 4498,86.1 \%)$, avoiding crowded spaces $(3517 / 4498,78.2 \%)$, and staying home when sick $(3857 / 4498,85.7 \%)$ all or most of the time (Table 3). Participants who reported only adhering to any of these NPIs sometimes, rarely, or never had higher odds of vaccine hesitancy. Participants who reported rarely or never wearing a face mask had an adjusted prevalence of vaccine hesitancy of 1.38 (95\% CI 1.22-1.56) compared with those who reported wearing a face mask all the time or most of the time. For physical distancing, those who reported adhering to this NPI sometimes had higher prevalence of vaccine hesitancy than those who practiced physical distancing all the time or most of the time (aPR 1.32, 95\% CI 1.18-1.48). Compared with those who reported avoiding crowded spaces all the time or most of the time, participants who reported rarely or never avoiding public spaces had higher prevalence of vaccine hesitancy (aPR $1.35,95 \%$ CI $1.21-1.50)$. 
Table 3. Associations between COVID-19 vaccine hesitancy, adherence to public health measures, and attitudes toward COVID-19 in October to November 2020 ( $\mathrm{N}=4498)$.

\begin{tabular}{lllllll}
\hline & \multicolumn{5}{c}{ Vaccine hesitancy, n (\%) } \\
Characteristic & Total, n (\%) & No & Yes & Prevalence ratio $^{\mathrm{a}}$ (95\% CI) & Adjusted prevalence ratio $^{\mathrm{b}}$ (95\% CI) \\
\hline Participants & $4498(100)$ & $1622(36.1)$ & $2876(63.9)$ & N/A $\mathrm{A}^{\mathrm{c}}$ & N/A
\end{tabular}

Adherence to nonpharmaceutical interventions

Physical distancing

$\begin{array}{llllll}\begin{array}{l}\text { All the time/most of the } \\ \text { time }\end{array} & 3777(84) & 1523(40.3) & 2254(69.7) & \operatorname{Ref}^{\mathrm{d}} & \text { Ref } \\ \text { Sometimes } & 457(10.2) & 68(14.9) & 389(85.1) & 1.43(1.28-1.59) & 1.32(1.18-1.48) \\ \text { Rarely/never } & 264(5.8) & 31(11.7) & 233(88.3) & 1.48(1.29-1.69) & 1.31(1.13-1.50)\end{array}$

Wearing face masks

$\begin{array}{llllll}\begin{array}{l}\text { All the time/most of the } \\ \text { time }\end{array} & 3868(86) & 1566(40.5) & 2302(69.5) & \text { Ref } & \text { Ref } \\ \text { Sometimes } & 274(6.1) & 36(13.1) & 238(86.9) & 1.46(1.28-1.67) & 1.34(1.16-1.54) \\ \text { Rarely/never } & 356(7.9) & 20(5.6) & 336(94.4) & 1.59(1.41-1.78) & 1.38(1.22-1.56)\end{array}$

\section{Avoiding crowded places}

All the time/most of the time

Sometimes

$457(10.2)$

$528(11.7)$

129 (28.2)

$2079(59.2) \quad$ Ref

Ref

Rarely/never

$59(11.2)$

$328(71.8) \quad 1.21(1.08-1.36)$

$1.16(1.03-1.31)$

Staying home when sick

All the time/most of the time

$3852(85.6) \quad 1505(39.1) \quad 2347(60.9) \quad$ Ref

$232(5.2) \quad 47(20.3)$

185 (79.7) $1.31(1.13-1.52)$

$1.35(1.21-1.50)$

Sometimes
Rarely/Never

$414(9.2) \quad 70(16.9)$

$344(83.1) \quad 1.36(1.22-1.53)$

$1.27(1.13-1.42)$

Attitudes toward COVID-19

Ever tested positive for COVID-19

$\begin{array}{llllll}\text { No } & 4385(97.5) & 1583(36.1) & 2802(63.9) & \text { Ref } & \text { Ref } \\ \text { Yes } & 113(2.5) & 39(34.5) & 74(65.5) & 1.02(0.81-1.29) & 1.05(0.83-1.32)\end{array}$

Know someone who had COVID-19

$\begin{array}{llllll}\text { No } & 3162(70.3) & 1101(34.8) & 2061(65.2) & \text { Ref } & \text { Ref } \\ \text { Yes } & 1336(29.7) & 521(39) & 815(61) & 0.94(0.86-1.01) & 0.98(0.90-1.06)\end{array}$

Anticipated effect of COVID-19 on own health

$\begin{array}{llllll}\text { Mild or no symptoms } & 1085(24.1) & 201(18.5) & 884(81.5) & \text { Ref } & \text { Ref } \\ \text { Manageable symptoms } & 1940(43.1) & 747(38.5) & 1193(61.5) & 0.75(0.69-0.82) & 0.80(0.73-0.86) \\ \text { Severe symptoms } & 1026(22.8) & 452(44.1) & 574(55.9) & 0.69(0.62-0.76) & 0.73(0.66-0.82) \\ \text { Possible death } & 447(9.9) & 222(49.7) & 225(50.3) & 0.62(0.53-0.72) & 0.65(0.56-0.76)\end{array}$

Concern for friends/family getting sick

$\begin{array}{llllll}\text { Not concerned } & 1204(26.8) & 165(13.7) & 1039(86.3) & \text { Ref } & \text { Ref } \\ \text { Concerned } & 3294(73.2) & 1457(44.2) & 1837(55.8) & 0.65(0.60-0.70) & 0.70(0.64-0.76)\end{array}$

Live with someone who is high risk for COVID-19

$\begin{array}{llllll}\text { No } & 2649(58.9) & 874(33) & 1775(67) & \text { Ref } & \text { Ref } \\ \text { Yes } & 1849(41.1) & 748(40.5) & 1101(59.5) & 0.89(0.82-0.96) & 0.89(0.83-0.97)\end{array}$

${ }^{\mathrm{a}}$ Prevalence ratio is the prevalence of vaccine hesitancy compared with the prevalence of planning to take a COVID-19 vaccine. 
${ }^{\mathrm{b}}$ Adjusted for sex, age, province of residence, household income, education level, ethnicity, and political leaning.

${ }^{\mathrm{c}} \mathrm{N} / \mathrm{A}$ : not applicable.

${ }^{\mathrm{d}}$ Ref: reference value.

\section{Attitudes Toward COVID-19}

A small proportion of participants had tested positive for COVID-19 (113/4498, 3\%) and almost one-third (1336/4498, $29.7 \%$ ) knew someone who had tested positive for COVID-19 (Table 3). Participants who were concerned about their friends or family getting sick from COVID-19 had lower prevalence of vaccine hesitancy compared with those who were not concerned (aPR 0.70, 95\% CI 0.64-0.76). Participants who reported living with an individual at high risk had lower prevalence (aPR $0.89,95 \%$ CI 0.83-0.97) of vaccine hesitancy. Compared with participants who anticipated experiencing mild or no symptoms in the event of contracting COVID-19, participants had lower prevalence of vaccine hesitancy if they reported they anticipated experiencing manageable symptoms (aPR 0.80, 95\% CI 0.73-0.86), severe symptoms (aPR 0.73, 95\% CI 0.66-0.82), or possible death (aPR 0.65 , 95\% CI $0.56-0.76)$.

\section{Trusted Sources of COVID-19 Information and Institutions}

Participants who trusted chief medical officers of health (aPR $0.54,95 \%$ CI $0.47-0.61$ ) and public health websites (aPR 0.68,
95\% CI 0.59-0.77) had lower prevalence of vaccine hesitancy compared with participants who did not (Table 4). Those who reported trusting internet searches for COVID-19 information had a higher prevalence of vaccine hesitancy compared to those who did not (aPR 1.34, 95\% CI 1.21-1.49). Participants who reported that their most trusted social media platform was Reddit (aPR 0.64, 95\% CI 0.51-0.80) had lower vaccine hesitancy than those who did not trust this source. We found that distrust in health care institutions, government, technology companies, finance industries, and professional services was associated with vaccine hesitancy (Table 4). Participants who reported that they did not trust government institutions had higher prevalence of vaccine hesitancy (aPR 1.61, 95\% CI 1.46-1.78) compared with those who reported trust in government institutions. The prevalence of hesitancy was also higher in those who did not trust health care (aPR 1.43, 95\% CI 1.25-1.62), technology (aPR $1.22,95 \%$ CI 1.08-1.38), and finance (aPR 1.14, 95\% CI 1.03-1.25) compared with those who reported trust in these institutions. 
Table 4. Associations between COVID-19 vaccine hesitancy, trusted sources of COVID-19 information, and trust in institutions in October to November $2020(\mathrm{~N}=4498)^{\mathrm{a}}$.

\begin{tabular}{|c|c|c|c|c|c|}
\hline \multirow[t]{2}{*}{ Sources } & \multirow[t]{2}{*}{ Total $(\mathrm{N})$} & \multicolumn{2}{|c|}{ Vaccine hesitancy, n (\%) } & \multirow[t]{2}{*}{ Prevalence ratio $^{\mathrm{b}}(95 \% \mathrm{CI})$} & \multirow[t]{2}{*}{ Adjusted prevalence ratio ${ }^{\mathrm{c}}(95 \% \mathrm{CI})$} \\
\hline & & No & Yes & & \\
\hline \multicolumn{6}{|c|}{ Most trusted sources for COVID-19 information } \\
\hline $\begin{array}{l}\text { Chief Medical Officer of } \\
\text { Health }\end{array}$ & 1933 & $904(46.8)$ & $1029(53.2)$ & $0.76(0.70-0.82)$ & $0.80(0.73-0.86)$ \\
\hline Public health websites & 1754 & $778(44.4)$ & $976(55.6)$ & $0.83(0.77-0.90)$ & $0.86(0.80-0.94)$ \\
\hline Health care provider & 1239 & $514(41.5)$ & $725(58.5)$ & $0.91(0.84-1.00)$ & $0.93(0.85-1.01)$ \\
\hline Television/radio news & 607 & $233(38.4)$ & $374(61.6)$ & $0.98(0.88-1.10)$ & $0.96(0.86-1.07)$ \\
\hline Internet searches (eg, Google) & 529 & $81(15.3)$ & $448(84.7)$ & $1.43(1.29-1.58)$ & $1.34(1.21-1.49)$ \\
\hline Friends and family & 159 & $33(20.8)$ & $126(79.2)$ & $1.28(1.07-1.53)$ & $1.16(0.97-1.39)$ \\
\hline Print newspaper & 134 & $55(41)$ & $79(59)$ & $0.94(0.75-1.18)$ & $0.96(0.77-1.21)$ \\
\hline \multicolumn{6}{|c|}{ Most trusted social media platforms for COVID-19 information } \\
\hline Facebook & 2167 & $778(35.9)$ & $1389(64.1)$ & $1.00(0.93-1.08)$ & $0.98(0.91-1.06)$ \\
\hline YouTube & 976 & $297(30.4)$ & $679(69.6)$ & $1.12(1.02-1.22)$ & $1.08(0.99-1.17)$ \\
\hline Twitter & 797 & $342(42.9)$ & $455(57.1)$ & $0.87(0.79-0.96)$ & $0.91(0.82-1.00)$ \\
\hline Instagram & 450 & $144(32)$ & $396(88)$ & $1.07(0.95-1.21)$ & $1.09(0.96-1.22)$ \\
\hline Reddit & 407 & $196(48.2)$ & $211(51.8)$ & $0.79(0.69-0.91)$ & $0.84(0.72-0.97)$ \\
\hline \multicolumn{6}{|l|}{ Trust in institutions } \\
\hline \multicolumn{6}{|l|}{ Health care } \\
\hline Trust & 3370 & $1440(42.7)$ & $1930(57.3)$ & $\operatorname{Ref}^{\mathrm{d}}$ & Ref \\
\hline Neutral & 828 & 154 (18.6) & $674(81.4)$ & $1.42(1.30-1.55)$ & $1.33(1.22-1.45)$ \\
\hline Do not trust & 300 & $28(9.3)$ & $272(90.7)$ & $1.58(1.39-1.80)$ & $1.43(1.25-1.62)$ \\
\hline \multicolumn{6}{|l|}{ Government } \\
\hline Trust & 1401 & $744(53.1)$ & 657 (46.9) & Ref & Ref \\
\hline Neutral & 1414 & $569(40.2)$ & $845(59.8)$ & $1.27(1.15-1.41)$ & $1.24(1.12-1.38)$ \\
\hline Do not trust & 1683 & $309(18.4)$ & 1374 (81.6) & $1.74(1.59-1.91)$ & $1.61(1.46-1.78)$ \\
\hline \multicolumn{6}{|l|}{ Technology } \\
\hline Trust & 579 & $261(45.1)$ & $318(54.9)$ & Ref & Ref \\
\hline Neutral & 1707 & $648(38)$ & $1059(62)$ & $1.13(1.00-1.28)$ & $1.15(1.01-1.30)$ \\
\hline Do not trust & 2212 & $713(32.2)$ & $1499(67.8)$ & $1.23(1.09-1.39)$ & $1.22(1.08-1.38)$ \\
\hline \multicolumn{6}{|l|}{ Finance } \\
\hline Trust & 1325 & $537(40.5)$ & $788(59.5)$ & Ref & Ref \\
\hline Neutral & 1824 & $641(35.1)$ & $1183(64.9)$ & $1.09(1.00-1.19)$ & $1.09(0.99-1.19)$ \\
\hline Do not trust & 1349 & 444 (32.9) & $905(67.1)$ & $1.12(1.03-1.24)$ & $1.14(1.03-1.25)$ \\
\hline
\end{tabular}

${ }^{\mathrm{a}}$ Participants could pick more than one most trusted source from each list.

${ }^{b}$ Prevalence ratio is the prevalence of vaccine hesitancy compared with the prevalence of planning to take a COVID-19 vaccine.

${ }^{c}$ Adjusted for sex, age, province of residence, household income, education level, ethnicity, and political leaning.

${ }^{\mathrm{d}}$ Ref: reference value.

\section{Cluster Analysis}

Three distinct nonoverlapping clusters were identified through cluster analysis (Multimedia Appendix 2). Cluster 1 (the vaccine and NPI-accepting cluster) consisted of 1652 (36.7\%) participants who reported willingness to take a COVID-19 vaccine and adherence to NPIs, including physical distancing, wearing a face mask in public spaces, staying home when sick, and avoiding public spaces. The 2099 (46.7\%) participants in Cluster 2 (the vaccine waiting and NPI accepting cluster) also reported adherence to NPIs but were vaccine hesitant, with 1652 $(78.7 \%)$ reporting that they would eventually get a vaccine; 
however, they planned to wait a while (Figure 1). Cluster 3 (the vaccine and NPI nonaccepting cluster) consisted of 747 (16.6\%) participants who reported less adherence to NPIs and were vaccine hesitant, with 557 (74.6\%) reporting that they would not take a COVID-19 vaccine when offered (Table 5).

Figure 1. Willingness of the survey participants, by cluster, to take the COVID-19 vaccine when available.

Cluster 3: Not Accepting

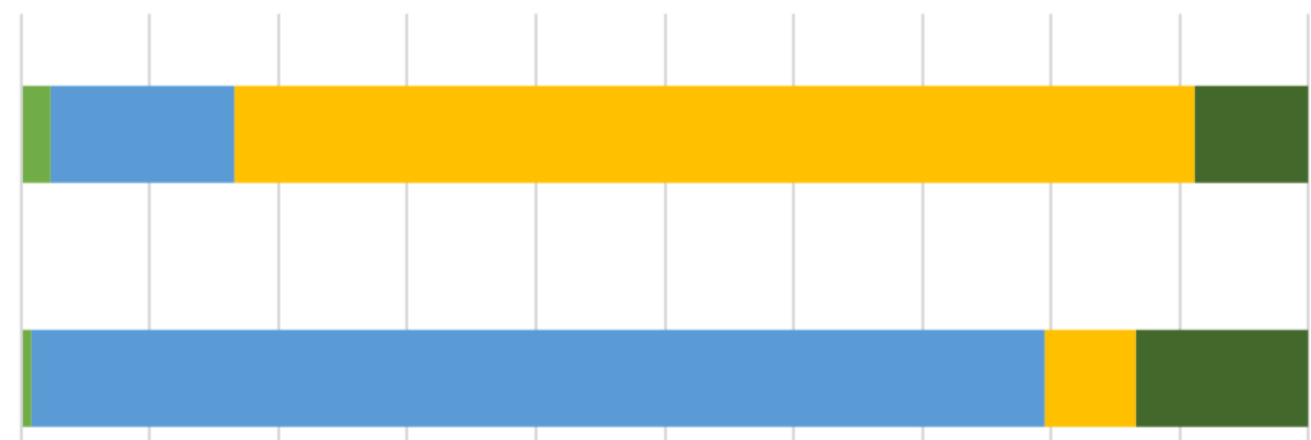

Cluster 2: Waiting

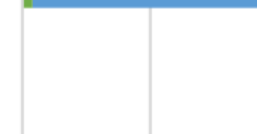

Cluster 1: Accepting

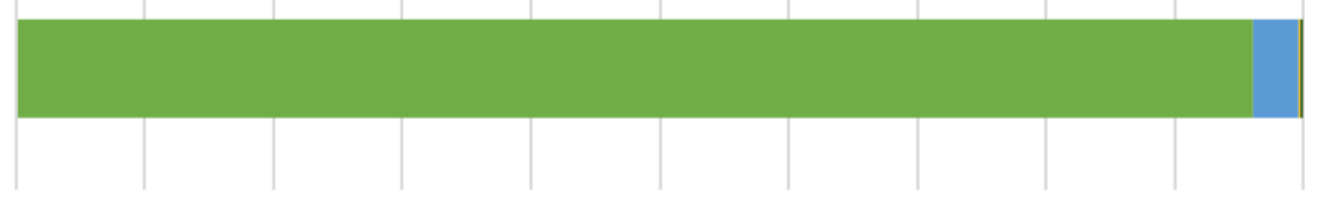

$0 \%$

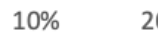

$20 \% \quad 30 \%$

$40 \%$

$50 \%$

$60 \%$

$70 \%$

$80 \%$

$90 \%$

$100 \%$

- Yes, As Soon As Possible

-Yes, Eventually $\quad$ No

- Not Sure 
Table 5. Participant characteristics by cluster (N=4498) in October to November 2020.

\begin{tabular}{|c|c|c|c|c|c|c|}
\hline \multirow[b]{2}{*}{ Characteristic } & \multirow[b]{2}{*}{ Total, n (\%) } & \multicolumn{3}{|c|}{ Cluster, n (\%) } & \multicolumn{2}{|c|}{ Prevalence ratio $^{\mathrm{a}}(95 \% \mathrm{CI})$} \\
\hline & & $\begin{array}{l}\text { Cluster 1: } \\
\text { Accepting }\end{array}$ & Cluster 2: Waiting & $\begin{array}{l}\text { Cluster 3: Not } \\
\text { accepting }\end{array}$ & $\begin{array}{l}\text { Cluster } 1 \text { versus } \\
\text { Cluster } 2\end{array}$ & $\begin{array}{l}\text { Cluster } 3 \text { versus } \\
\text { Cluster } 2\end{array}$ \\
\hline Participants & $4498(100)$ & $1652(36.7)$ & 2099 (46.7) & $747(16.6)$ & $\mathrm{N} / \mathrm{A}^{\mathrm{b}}$ & N/A \\
\hline \multicolumn{7}{|l|}{ Sex at birth } \\
\hline Female & $2294(51)$ & $837(36.5)$ & $1171(51)$ & $286(12.5)$ & $\operatorname{Ref}^{c}$ & Ref \\
\hline Male & $2204(49)$ & $815(37)$ & $928(42.1)$ & $461(20.9)$ & $1.12(1.02-1.24)$ & $1.69(1.46-1.96)$ \\
\hline \multicolumn{7}{|l|}{ Age (years) } \\
\hline $18-34$ & $1341(29.8)$ & $511(38.1)$ & $621(46.3)$ & $209(15.6)$ & Ref & Ref \\
\hline $35-54$ & $1585(35.2)$ & $516(32.5)$ & $741(46.8)$ & $328(20.7)$ & $0.91(0.80-1.03)$ & $1.22(1.02-1.45)$ \\
\hline $55+$ & $1572(34.9)$ & $625(39.8)$ & 737 (46.9) & $210(13.3)$ & $1.02(0.890-1.14)$ & $0.88(0.73-1.06)$ \\
\hline \multicolumn{7}{|l|}{ Province of residence } \\
\hline Alberta & $1998(44.4)$ & $684(34.2)$ & $836(41.8)$ & 478 (23.9) & Ref & Ref \\
\hline British Columbia & $502(11.2)$ & $185(36.9)$ & $273(54.4)$ & $44(8.8)$ & $0.90(0.76-1.06)$ & $0.38(0.28-0.52)$ \\
\hline Prairie provinces ${ }^{\mathrm{d}}$ & 445 (9.9) & $158(35.5)$ & $215(48.3)$ & $72(16.2)$ & $0.94(0.79-1.12)$ & $0.69(0.54-0.88)$ \\
\hline Ontario & $800(17.8)$ & $318(39.7)$ & $404(50.5)$ & $78(9.8)$ & $0.98(0.86-1.12)$ & $0.44(0.35-0.57)$ \\
\hline Quebec & $502(11.2)$ & $202(40.2)$ & $250(49.8)$ & $50(10)$ & $0.99(0.85-1.16)$ & $0.46(0.34-0.61)$ \\
\hline Atlantic provinces ${ }^{\mathrm{d}}$ & $251(5.6)$ & $105(41.8)$ & $121(48.2)$ & $25(10)$ & $1.03(0.84-1.27)$ & $0.47(0.31-0.70)$ \\
\hline \multicolumn{7}{|l|}{ Household income $^{\mathrm{e}}$ (CAD) } \\
\hline$<\$ 50,000$ & $1030(22.9)$ & $341(33.1)$ & $532(51.7)$ & $157(15.2)$ & Ref & Ref \\
\hline$\$ 50,000-\$ 99,999$ & $1353(30.1)$ & $489(36.1)$ & $644(47.6)$ & $220(16.3)$ & $1.10(0.96-1.27)$ & $1.12(0.91-1.37)$ \\
\hline$\$ 100,000-\$ 199,999$ & $1300(28.9)$ & $532(40.9)$ & $559(43)$ & $209(16.1)$ & $1.25(1.09-1.43)$ & $1.19(0.97-1.47)$ \\
\hline$\geq \$ 200,000$ & $229(5.1)$ & $100(43.7)$ & $87(38)$ & $42(18.3)$ & $1.37(1.10-1.71)$ & $1.43(1.02-2.01)$ \\
\hline Rather not say & $586(13)$ & $190(32.4)$ & $277(47.3)$ & $119(20.3)$ & $1.04(0.87-1.24)$ & $1.32(1.04-1.67)$ \\
\hline \multicolumn{7}{|l|}{ Highest level of education } \\
\hline High school graduate or less & 897 (19.9) & $262(29.2)$ & $433(48.3)$ & $202(22.5)$ & Ref & Ref \\
\hline Some college or trade school & $840(18.7)$ & $240(28.6)$ & $421(50.1)$ & $179(21.3)$ & $0.96(0.81-1.15)$ & $0.94(0.77-1.15)$ \\
\hline College or trade school & $996(22.1)$ & $304(30.5)$ & $491(49.3)$ & $201(20.2)$ & $1.01(0.86-1.20)$ & $0.91(0.75-1.11)$ \\
\hline Some university & $454(10.1)$ & $190(41.8)$ & $197(43.4)$ & $67(14.8)$ & $1.30(1.08-1.57)$ & $0.80(0.61-1.05)$ \\
\hline University degree & $1311(29.2)$ & $656(50)$ & $557(42.5)$ & $98(7.5)$ & $1.43(1.24-1.66)$ & $0.47(0.37-0.60)$ \\
\hline \multicolumn{7}{|l|}{ Ethnicity } \\
\hline Caucasian & $3862(85.9)$ & $1455(37.7)$ & $1765(45.7)$ & $642(16.6)$ & Ref & Ref \\
\hline Indigenous/First Nations/Metis/Inuit & $228(5.1)$ & $71(31.1)$ & $107(46.9)$ & $50(21.9)$ & $0.88(0.70-1.12)$ & $1.19(0.90-1.59)$ \\
\hline Asian & $193(4.3)$ & $67(34.7)$ & $115(59.6)$ & $11(5.7)$ & $0.81(0.64-1.04)$ & $0.33(0.18-0.59)$ \\
\hline Caribbean/African/South American & $70(1.5)$ & $16(22.9)$ & $43(61.4)$ & $11(15.7)$ & $0.60(0.37-0.98)$ & $0.76(0.42-1.39)$ \\
\hline Other & $145(3.2)$ & $43(29.6)$ & $69(47.6)$ & $33(22.8)$ & $0.85(0.63-1.15)$ & $1.21(0.85-1.72)$ \\
\hline \multicolumn{7}{|l|}{ Political leaning } \\
\hline Liberal & $1841(40.9)$ & $971(52.7)$ & $821(44.6)$ & $49(2.7)$ & $1.48(1.30-1.68)$ & $0.29(0.21-0.40)$ \\
\hline Moderate/middle of the road & $1029(22.9)$ & $327(31.8)$ & $565(54.9)$ & $137(13.3)$ & Ref & Ref \\
\hline Conservative & $1628(36.2)$ & $354(21.7)$ & $713(43.8)$ & $561(34.5)$ & $0.91(0.78-1.05)$ & $2.26(1.87-2.72)$ \\
\hline
\end{tabular}

${ }^{\mathrm{a}}$ Determined using negative binomial regression.

${ }^{\mathrm{b}} \mathrm{N} / \mathrm{A}$ : not applicable. 
${ }^{\mathrm{c}}$ Ref: reference value.

${ }^{\mathrm{d}}$ Prairie provinces include Saskatchewan and Manitoba; Atlantic provinces include Nova Scotia, New Brunswick, Prince Edward Island, and Newfoundland and Labrador.

${ }^{\mathrm{e}} \mathrm{CAD} \$ 1=\mathrm{US} \$ 0.75$.

Compared with participants in Cluster 2 (the vaccine waiting and NPI-accepting cluster), participants in Cluster 3 (the vaccine and NPI nonaccepting cluster) were more likely to be male (PR $1.69,95 \%$ CI 1.46-1.96), 35-54 years of age (PR 1.22, 95\% CI 1.02-1.45), have a household income of CAD \$200,000 (USD $\$ 150,200$; CAD $\$ 1=\mathrm{US} \$ 0.75$ ) or more (PR 1.32, 95\% CI 1.04-1.67), and report a conservative political leaning (PR 2.26, 95\% CI 1.87-2.72). Participants in Cluster 1 (the vaccine and NPI-accepting cluster) were more likely to be Liberal leaning (PR 1.48, 95\% CI 1.30-1.68), have some university education (PR 1.30, 95\% CI 1.08-1.57), or a university degree (PR 1.43, 95\% CI 1.24-1.66), have an annual household income of CAD $\$ 100,000-\$ 199,999$ (PR 1.25, 95\% CI 1.09-1.43) or CAD $\$ 200,000$ or more (PR 1.37, 95\% CI 1.10-1.71), and male (PR $1.12,95 \%$ CI 1.02-1.24) compared with participants in Cluster 2 (the vaccine-waiting and NPI-accepting cluster).

\section{Discussion}

\section{Principal Findings}

In this national cross-sectional survey completed in the fall of 2020 prior to the approval of COVID-19 vaccines in Canada, we found that $63.9 \%(2876 / 4498)$ of the participants reported COVID-19 vaccine hesitancy, ranging from delaying vaccine administration when offered to not planning to take a vaccine. Vaccine hesitancy was associated with several sociodemographic factors including (1) younger age (18-39 years), (2) lower education, and (3) non-Liberal political leaning. Participants who reported vaccine hesitancy had higher prevalence of reporting being concerned about vaccine side effects, did not believe that a COVID-19 vaccine would end the pandemic or that the benefits of a COVID-19 vaccine outweighed the risks, and had lower prevalence of reporting being influenced by peers or health care professionals. We identified 3 distinct participant clusters: (1) participants who reported adherence to NPIs and did not have vaccine hesitancy, (2) individuals who reported adherence to NPIs but did have vaccine hesitancy, and (3) individuals who reported less adherence to NPIs and vaccine hesitancy.

The 3 distinct clusters of vaccine acceptance can inform targeted vaccination campaign messaging in a novel way by directing messages to address cluster-specific concerns with respect to vaccine hesitancy. The majority of the participants in Cluster 2 (the vaccine waiting and NPI-accepting cluster) planned to delay taking a vaccine when offered, while the majority in Cluster 3 (the vaccine and NPI nonaccepting cluster) did not intend to take a vaccine at all. Messaging related to preventing COVID among friends and family, highlighting the benefits, and ensuring health care workers are knowledgeable and supported in their vaccine counselling may be more helpful for those in Cluster 2 relative to those in Cluster 3. Participants in Cluster 3 were more likely to be male, $35-54$ years of age, have an annual household income of CAD $\$ 200,000$ or more, report Conservative political leaning, and live in Alberta compared with participants in Cluster 2. The characteristics of Cluster 3 are consistent with current trends in vaccine uptake in that less uptake has been seen among Albertans, males, and individuals aged 18 to 59 years as of October 23, 2021 [17]. Based on our findings, Cluster 3 appears quite challenging to target messaging toward and further qualitative research is needed to determine how best to target this subgroup of vaccine-hesitant individuals to increase vaccine uptake.

As of October 27, 2021, more than 1,700,000 Canadians have been infected with COVID-19 and more than 28,000 Canadians have died [1]. Reported intention to get vaccinated has been variable [18-23], and as supply of vaccine outweighs demand among eligible individuals within Canada [24], there are growing concerns about vaccine hesitancy with respect to COVID-19 vaccines. The Government of Canada reports that as of October 23, 2021, 29,613,930 (77\%) individuals 12 years of age or older had received 1 dose of COVID-19 vaccine and $28,086,337(73 \%)$ were fully vaccinated [17]. Vaccine hesitancy among Canadians has decreased since the time our survey was administered, which is likely multifactorial. A recent qualitative study in the United States found that vaccine uptake among individuals who were initially vaccine hesitant is related to 3 factors: (1) intrinsic factors (eg, protecting oneself from COVID-19), (2) extrinsic factors (eg, protecting family or friends), and (3) structural factors (eg, vaccine mandates) [25].

While there has been a decrease in vaccine hesitancy over time, many of the underlying predictors of hesitancy have remained stable over time [5,7,10,26-29]. Many studies [10,19-21,30,31] have reported that female sex at birth was associated with COVID-19 vaccine hesitancy [18]. We found that lower education level was associated with COVID-19 vaccine hesitancy. Both low [10,19,20,30] and high [32] education level have been associated with COVID-19 vaccine hesitancy, while lower household income has more consistently been associated with vaccine hesitancy $[10,18,30]$. The conflicting associations between these sociodemographic factors and vaccine hesitancy suggest that these associations may be region-specific on a global scale as was identified by Lazarus et al [32] or may be time-dependent, as these cross-sectional surveys were completed at different points of time in the COVID-19 pandemic.

We did not find an association between ethnicity and COVID-19 vaccine hesitancy, although an association has been reported in several other studies [19,29,30,33]. In a qualitative study, Momplaisir et al [33] found several themes that contributed to vaccine hesitancy among individuals who identified as Black, including mistrust in the medical community, racial injustice, and COVID-19-specific concerns, including the speed of development and concerns about potential side effects. This highlights that COVID-19 vaccine hesitancy is complex with many contributing factors, all of which need to be addressed to effectively combat vaccine hesitancy and encourage individuals to take a COVID-19 vaccine when offered. Although population segments that are more likely to be vaccine hesitant can be 
identified and messages can be tailored to those population segments, the content and delivery of the messaging needs to consider the complex interaction of all the domains of the SAGE working group vaccine hesitancy determinant framework (ie, contextual influences, vaccine characteristics, and individual/social group influences) [8]. Messaging needs to be designed in collaboration with these population segments through partnership-based community-embedded work to address the complex and unique circumstances contributing to vaccine hesitancy.

The influences of COVID-19 vaccine characteristics and administration of COVID-19 vaccines on vaccine hesitancy are unique compared to annual influenza campaigns or childhood immunization schedules. In response to the COVID-19 pandemic, the scientific community has come together to develop safe and effective vaccines [2]. At the time of survey administration, prior to the regulatory approval of COVID-19 vaccines in Canada, we found that almost half of the respondents were concerned that these vaccines had been developed too quickly and the majority were concerned about the short- and long-term vaccine side effects. These concerns about COVID-19 vaccines have been reported in other studies $[19,31,33]$, and we found that they were associated with vaccine hesitancy. We also found that COVID-19 vaccine hesitancy was associated with lower concern about the consequences of becoming infected with COVID-19 or concern about family or friends becoming infected. Vaccination campaigns need to address these COVID-19-specific factors in their messaging.

Trust in government has been identified as a factor associated with acceptance of a COVID-19 vaccine [18]. Consistent with this, we found that vaccine hesitancy was associated with a lack of trust in government and health care institutions. When developing messaging to combat COVID-19 vaccine hesitancy, it is important to consider the importance of trust, which has been highlighted in previous pandemics, including the H1N1 pandemic [34]. To improve trust and consistency of messaging, supportive programs need to be available for health care workers to build knowledge and confidence in their messaging. The trusted sources of COVID-19 information should also be considered when designing targeted vaccination campaigns.

\section{Limitations}

The major limitation of this cross-sectional study was that it represents one snapshot in time in the fall of 2020 prior to the approval of COVID-19 vaccines in Canada and as the country was entering the second wave of the pandemic; therefore, the responses provided by participants at that time have evolved. The survey recruited participants from an existing voluntary nationwide panel designed to be representative of the Canadian population; however, by using a panel, there will be a component of selection bias as participants have volunteered to partake in research surveys through an electronic platform, which may lead to increased selection of individuals with higher socioeconomic status or education level leading to an underestimation of vaccine hesitancy. We included all provinces and territories in our sampling strata; however, we did oversample Alberta, which could lead to bias in the results and make these findings less generalizable to the Canadian population. To minimize this bias, province of residence was included in all adjusted analyses. Response bias should also be considered as individuals who chose to respond to the web-based survey may differ systematically from those who chose not to respond.

\section{Conclusion}

COVID-19 vaccines are an important tool in the fight against the COVID-19 pandemic; yet, vaccine hesitancy is a concern. We have identified population segments that are associated with vaccine hesitancy (eg, younger age, lower education level) that can be targeted with public health messaging as well as attitudes toward COVID-19, COVID-19 vaccines, and NPIs that can inform messaging content. Effectively addressing vaccine hesitancy is important to increase vaccine uptake.

\section{Acknowledgments}

This study was funded by a COVID-19 Rapid Response Funding Grant from Alberta Innovates (grant 202100489).

\section{Authors' Contributions}

JLB, RL, OA, BS, HS, MM, MMF, TT, BJM, DAM, JH, and RJO were all involved in study design. RL, JLB, BS, HS, MM, MMF, TT, BJM, DAM, JH, and RJO developed the survey. JLB, OA, BS, and RJO performed the analysis. JLB, OA, and RL wrote the initial draft of the paper. All authors reviewed the final manuscript.

\section{Conflicts of Interest}

None declared.

\section{Multimedia Appendix 1}

Survey questions.

[DOCX File, 987 KB-Multimedia Appendix 1]

\section{Multimedia Appendix 2}

Cluster analysis. 


\section{References}

1. Coronavirus disease (COVID-19): Outbreak update. Government of Canada. URL: https://www.canada.ca/en/public-health/ services/diseases/2019-novel-coronavirus-infection.html [accessed 2021-09-28]

2. Vaccines for COVID-19: Approved vaccines. Government of Canada. URL: https://www.canada.ca/en/health-canada/ services/drugs-health-products/covid19-industry/drugs-vaccines-treatments/vaccines.html [accessed 2021-04-03]

3. Coronavirus disease (COVID-19): Prevention and risks. Government of Canada. URL: https://www.canada.ca/en/ public-health/services/diseases/2019-novel-coronavirus-infection/prevention-risks/measures-reduce-community.html [accessed 2020-12-10]

4. Benham JL, Lang R, Kovacs Burns K, MacKean G, Léveillé T, McCormack B, et al. Attitudes, current behaviours and barriers to public health measures that reduce COVID-19 transmission: A qualitative study to inform public health messaging. PLoS One 2021;16(2):e0246941 [FREE Full text] [doi: 10.1371/journal.pone.0246941] [Medline: 33606782]

5. Lang R, Benham JL, Atabati O, Hollis A, Tombe T, Shaffer B, et al. Attitudes, behaviours and barriers to public health measures for COVID-19: a survey to inform public health messaging. BMC Public Health 2021 Apr 21;21(1):765 [FREE Full text] [doi: 10.1186/s12889-021-10790-0] [Medline: $\underline{\text { 33882896] }}$

6. Zizzo J. The Missing Link in the Covid-19 Vaccine Race. Hum Vaccin Immunother 2021 May 04;17(5):1326-1328 [FREE Full text] [doi: 10.1080/21645515.2020.1831859] [Medline: 33079612]

7. Lin C, Tu P, Beitsch LM. Confidence and Receptivity for COVID-19 Vaccines: A Rapid Systematic Review. Vaccines (Basel) 2020 Dec 30;9(1):16 [FREE Full text] [doi: 10.3390/vaccines9010016] [Medline: 33396832]

8. MacDonald NE, SAGE Working Group on Vaccine Hesitancy. Vaccine hesitancy: Definition, scope and determinants. Vaccine 2015 Aug 14;33(34):4161-4164 [FREE Full text] [doi: 10.1016/j.vaccine.2015.04.036] [Medline: 25896383]

9. Larson HJ, Jarrett C, Eckersberger E, Smith DMD, Paterson P. Understanding vaccine hesitancy around vaccines and vaccination from a global perspective: a systematic review of published literature, 2007-2012. Vaccine 2014 Apr 17;32(19):2150-2159. [doi: 10.1016/j.vaccine.2014.01.081] [Medline: 24598724]

10. Cascini F, Pantovic A, Al-Ajlouni Y, Failla G, Ricciardi W. Attitudes, acceptance and hesitancy among the general population worldwide to receive the COVID-19 vaccines and their contributing factors: A systematic review. EClinicalMedicine 2021 Oct;40:101113 [FREE Full text] [doi: 10.1016/j.eclinm.2021.101113] [Medline: $\underline{34490416}$ ]

11. Salomoni MG, Di Valerio Z, Gabrielli E, Montalti M, Tedesco D, Guaraldi F, et al. Hesitant or Not Hesitant? A Systematic Review on Global COVID-19 Vaccine Acceptance in Different Populations. Vaccines (Basel) 2021 Aug 06;9(8):873 [FREE Full text] [doi: 10.3390/vaccines9080873] [Medline: $\underline{34452000]}$

12. About the institute. Angus Reid Institute. URL: http://angusreid.org/about ari/ [accessed 2021-01-11]

13. von Elm E, Altman DG, Egger M, Pocock SJ, Gøtzsche PC, Vandenbroucke JP, STROBE Initiative. The Strengthening the Reporting of Observational Studies in Epidemiology (STROBE) statement: guidelines for reporting observational studies. Ann Intern Med 2007 Oct 16;147(8):573-577 [FREE Full text] [doi: 10.7326/0003-4819-147-8-200710160-00010] [Medline: 17938396 ]

14. Syan SK, Gohari MR, Levitt EE, Belisario K, Gillard J, DeJesus J, et al. COVID-19 Vaccine Perceptions and Differences by Sex, Age, and Education in 1,367 Community Adults in Ontario. Front Public Health 2021;9:719665 [FREE Full text] [doi: 10.3389/fpubh.2021.719665] [Medline: 34631647]

15. Nehal KR, Steendam LM, Campos Ponce M, van der Hoeven M, Smit GSA. Worldwide Vaccination Willingness for COVID-19: A Systematic Review and Meta-Analysis. Vaccines (Basel) 2021 Sep 24;9(10):1071 [FREE Full text] [doi: 10.3390/vaccines9101071] [Medline: 34696179]

16. Kachurka R, Krawczyk M, Rachubik J. Persuasive Messages Will Not Increase COVID-19 Vaccine Acceptance: Evidence from a Nationwide Online Experiment. Vaccines (Basel) 2021 Sep 30;9(10):1113 [FREE Full text] [doi: 10.3390/vaccines9101113] [Medline: 34696221]

17. COVID-19 vaccination in Canada. Government of Canada. URL: https://health-infobase.canada.ca/covid-19/ vaccination-coverage/ [accessed 2021-11-05]

18. Lazarus JV, Ratzan SC, Palayew A, Gostin LO, Larson HJ, Rabin K, et al. A global survey of potential acceptance of a COVID-19 vaccine. Nat Med 2021 Feb;27(2):225-228 [FREE Full text] [doi: 10.1038/s41591-020-1124-9] [Medline: $\underline{33082575]}$

19. Reiter PL, Pennell ML, Katz ML. Acceptability of a COVID-19 vaccine among adults in the United States: How many people would get vaccinated? Vaccine 2020 Sep 29;38(42):6500-6507 [FREE Full text] [doi: 10.1016/j.vaccine.2020.08.043] [Medline: 32863069]

20. Schwarzinger M, Watson V, Arwidson P, Alla F, Luchini S. COVID-19 vaccine hesitancy in a representative working-age population in France: a survey experiment based on vaccine characteristics. The Lancet Public Health 2021 Apr;6(4):e210-e221. [doi: 10.1016/s2468-2667(21)00012-8]

21. Detoc M, Bruel S, Frappe P, Tardy B, Botelho-Nevers E, Gagneux-Brunon A. Intention to participate in a COVID-19 vaccine clinical trial and to get vaccinated against COVID-19 in France during the pandemic. Vaccine 2020 Oct 21;38(45):7002-7006 [FREE Full text] [doi: 10.1016/j.vaccine.2020.09.041] [Medline: $\underline{32988688}$ ] 
22. Kwok K, Li K, Wei W, Tang A, Wong S, Lee S. Editor's Choice: Influenza vaccine uptake, COVID-19 vaccination intention and vaccine hesitancy among nurses: A survey. Int J Nurs Stud 2021 Feb;114:103854 [FREE Full text] [doi: 10.1016/j.ijnurstu.2020.103854] [Medline: 33326864]

23. Latkin CA, Dayton L, Yi G, Colon B, Kong X. Mask usage, social distancing, racial, and gender correlates of COVID-19 vaccine intentions among adults in the US. PLoS One 2021;16(2):e0246970 [FREE Full text] [doi:

10.1371/journal.pone.0246970] [Medline: 33592035]

24. Vaccines for COVID-19: Canada's vaccine supply. Government of Canada. URL: https://www.canada.ca/en/public-health/ services/diseases/2019-novel-coronavirus-infection/prevention-risks/covid-19-vaccine-treatment/vaccine-rollout.html [accessed 2021-04-03]

25. Moore R, Purvis RS, Hallgren E, Willis DE, Hall S, Reece S, et al. Motivations to Vaccinate Among Hesitant Adopters of the COVID-19 Vaccine. J Community Health 2021 Oct 23:1-9 [FREE Full text] [doi: 10.1007/s10900-021-01037-5] [Medline: $\underline{34687388]}$

26. Parsons Leigh J, Fiest K, Brundin-Mather R, Plotnikoff K, Soo A, Sypes EE, et al. A national cross-sectional survey of public perceptions of the COVID-19 pandemic: Self-reported beliefs, knowledge, and behaviors. PLoS One 2020;15(10):e0241259 [FREE Full text] [doi: 10.1371/journal.pone.0241259] [Medline: 33095836]

27. Fisher KA, Bloomstone SJ, Walder J, Crawford S, Fouayzi H, Mazor KM. Attitudes Toward a Potential SARS-CoV-2 Vaccine : A Survey of U.S. Adults. Ann Intern Med 2020 Dec 15;173(12):964-973 [FREE Full text] [doi: 10.7326/M20-3569] [Medline: $\underline{\text { 32886525] }}$

28. Mondal P, Sinharoy A, Su L. Sociodemographic predictors of COVID-19 vaccine acceptance: a nationwide US-based survey study. Public Health 2021 Sep;198:252-259 [FREE Full text] [doi: 10.1016/j.puhe.2021.07.028] [Medline: 34492505]

29. Ruiz JB, Bell RA. Predictors of intention to vaccinate against COVID-19: Results of a nationwide survey. Vaccine 2021 Feb 12;39(7):1080-1086 [FREE Full text] [doi: 10.1016/j.vaccine.2021.01.010] [Medline: 33461833]

30. Kreps S, Prasad S, Brownstein JS, Hswen Y, Garibaldi BT, Zhang B, et al. Factors Associated With US Adults' Likelihood of Accepting COVID-19 Vaccination. JAMA Netw Open 2020 Oct 01;3(10):e2025594 [FREE Full text] [doi: 10.1001/jamanetworkopen.2020.25594] [Medline: 33079199]

31. Dror AA, Eisenbach N, Taiber S, Morozov NG, Mizrachi M, Zigron A, et al. Vaccine hesitancy: the next challenge in the fight against COVID-19. Eur J Epidemiol 2020 Aug;35(8):775-779. [doi: 10.1007/s10654-020-00671-y] [Medline: 32785815]

32. Lazarus JV, Wyka K, Rauh L, Rabin K, Ratzan S, Gostin LO, et al. Hesitant or Not? The Association of Age, Gender, and Education with Potential Acceptance of a COVID-19 Vaccine: A Country-level Analysis. J Health Commun 2020 Oct 02;25(10):799-807. [doi: 10.1080/10810730.2020.1868630] [Medline: 33719881]

33. Momplaisir F, Haynes N, Nkwihoreze H, Nelson M, Werner RM, Jemmott J. Understanding Drivers of Coronavirus Disease 2019 Vaccine Hesitancy Among Blacks. Clin Infect Dis 2021 Nov 16;73(10):1784-1789 [FREE Full text] [doi: 10.1093/cid/ciab102] [Medline: $\underline{33560346]}$

34. Siegrist M, Zingg A. The Role of Public Trust During Pandemics. European Psychologist 2014 Jan 01;19(1):23-32. [doi: $\underline{10.1027 / 1016-9040 / a 000169]}$

\section{Abbreviations}

aPR: adjusted prevalence ratio

NPI: nonpharmacologic intervention

PR: prevalence ratio

SAGE: Strategic Advisory Group of Experts

STROBE: Strengthening Reporting of Observational Studies in Epidemiology

Edited by T Sanchez; submitted 13.05.21; peer-reviewed by P Valente, C Jacob; comments to author 05.08.21; revised version received 07.10.21; accepted 11.11.21; published 23.12.21

Please cite as:

Benham JL, Atabati O, Oxoby RJ, Mourali M, Shaffer B, Sheikh H, Boucher JC, Constantinescu C, Parsons Leigh J, Ivers NM, Ratzan $S C$, Fullerton MM, Tang T, Manns BJ, Marshall DA, Hu J, Lang $R$

COVID-19 Vaccine-Related Attitudes and Beliefs in Canada: National Cross-sectional Survey and Cluster Analysis

JMIR Public Health Surveill 2021;7(12):e30424

URL: https://publichealth.jmir.org/2021/12/e30424

doi: $10.2196 / 30424$

PMID: $\underline{34779784}$ 
(CJamie L Benham, Omid Atabati, Robert J Oxoby, Mehdi Mourali, Blake Shaffer, Hasan Sheikh, Jean-Christophe Boucher, Cora Constantinescu, Jeanna Parsons Leigh, Noah M Ivers, Scott C Ratzan, Madison M Fullerton, Theresa Tang, Braden J Manns, Deborah A Marshall, Jia Hu, Raynell Lang. Originally published in JMIR Public Health and Surveillance (https://publichealth.jmir.org), 23.12.2021. This is an open-access article distributed under the terms of the Creative Commons Attribution License (https://creativecommons.org/licenses/by/4.0/), which permits unrestricted use, distribution, and reproduction in any medium, provided the original work, first published in JMIR Public Health and Surveillance, is properly cited. The complete bibliographic information, a link to the original publication on https://publichealth.jmir.org, as well as this copyright and license information must be included. 